\title{
Adaptive E-learning using Deterministic Finite Automata
}

\author{
Pratima Sarkar \\ Assistant Professor \\ Sikkim Manipal Inastitute of Technology \\ Majhitar, East Sikkim
}

\author{
Chinmoy Kar \\ Assistant Professor \\ Sikkim Manipal Inastitute of Technology \\ Majhitar, East Sikkim
}

\begin{abstract}
Adaptive E-learning refers to adapt the way of presentation of educational material according to the student's needs. Understanding ability differs by student to student so the learning path should vary according to their understanding ability. Some students may understand by once some may needs more with different way. This paper represents a same topic with various approaches to the different classes of students with different understanding ability. The proposed approach in this paper is based on two concepts Deterministic Finite Automata (DFA) and Case Based Study (CBS), out of which DFA used for providing adaptive nature and shows the state transition according to their performance. Path of leaning varies with different student for a particular topic, this feature used to provide adaptively nature in E-learning. CBS used for providing study material based on the state. CBS uses case library to decide the study material.
\end{abstract}

\section{General Terms}

E-learning

Keywords: Adaptive E-learning, Deterministic finite automata (DFA).

\section{INTRODUCTION}

E-learning is the use of technology to enable people to learn anytime and anywhere. E- Learning can include training, the delivery of just-in-time information and guidance from experts. One of the major benefits of e-learning is learner autonomy. Platforms by offering courses tailored to the results, behaviors, tastes of learners, unconsciously. Elearning will improve the use of Educational material in web form in a Web-based educational system makes learning more popular. It motivates learners to explore alternative navigational paths through the domain knowledge and from different resources around the globe. In our educational system not possible to care each and every student separately but e-learning system provides special care for each student according to their capacity. For implementing E-learning this paper uses Deterministic Finite Automata (DFA) it helps in adaptive learning. Unless student totally prepared about the particular chapter they taught same topic different way. It also takes care of student's confidence by showing each time improvement. For each chapter different DFA is used. For weaker students it needs more transition to reach the goal or final state and for fast learners two transition are enough

The rest of this paper is organized as follows: Section 2 describes the related literatures review, and this paper presents the proposed solution in Section 3. This is followed by a description of the test case with corresponding DFA in Section 4. Finally, conclusion and future scope mentioned in Section 5.

\section{RELATED WORK}

Learning materials should match students' learning styles i.e. visual, auditory and kinesthetic either globally or sequentially [1]. For solving above problem paper [1] identifies the student's learning styles tendency through a set of questionnaire and questionnaire scores are used by the system as basis to provide the student a presentation of learning materials differently.

Characteristics of learner and ended up with rigid e-learning systems [2]. In paper [2] uses customized Learning Objects which adapts to learner's perspective is a powerful constraint of any e-learning system and this paper concentrates on learner's characteristics which include the learner's knowledge level and learning style.

Students are not homogeneous elements acting according to similar simple rules, but heterogeneous agents with complex behavioral rules and constraints. Three of the main differences are in their skill, knowledge, and dedication [3]. A student filtering mechanism and clustering technique is used to classify students that improve the results when using students in swarm intelligence-based scenario (Ant Colony Optimization).

The learning scheme generation algorithm is designed to be genetic. This is because the various learners' context parameter values are viewed as constraints to be fulfilled in the learning scheme generation and genetic algorithms are best suitable for handling multiple constraint satisfaction problems which have many alternative solutions [4].

In a basic e-learning scenario, the organization of course is normally the responsibility of the instructor of the course, while the students limit themselves to the consumption of the resources. By applying ant colony based methods to elearning scenarios, the interaction of every student with the elearning platform can also be used to improve the organization of the course [6].

Pedagogic Suggestions [10] the emphasis on pedagogic structure and conditioning the website's presentation based on Ant Colony Optimization. It uses a navigation graph where nodes are exercises or lessons and arcs are hypertext links. Graph is gradually modified through the release and evaporation of virtual pheromones that reflect the successes and failures of students roaming around the graph.

[8] is also uses Ant Colony optimization to determine the structure of the e-learning material is represented by a graph with valued arcs whose weights are optimized by virtual ants or students that release virtual pheromones along their paths. This gradual modification of the graph's structure improves its pedagogic pertinence in order to increase pedagogic success. 


\section{PROPOSED SOLUTION}

Most of the above state of art says about curriculum sequencing of a particular subject. In this paper assumption is sequencing of curriculum done by subject expert and concentrates on skill, knowledge, and dedication of the students [3] to decide learning path. This paper uses Deterministic Finite Automata (DFA) for implementing ELearning. In this solution for different Chapter of subject have a separate DFA. Deterministic Finite Automata has a fixed number of states and student can only be in one state at a time. In DFA transition of the states depends upon input and state. DFA can be represented by 5 -tuples $\left(\mathrm{Q}, \Sigma, \delta, \mathrm{q}_{0}, \mathrm{~F}\right)$ where

- $\quad \mathrm{Q}$ is a finite set of states.

- $\quad \Sigma$ is a finite set of input symbols called the alphabet

- $\delta$ is a transition function $(\delta: Q \times \Sigma \rightarrow Q)$

- $\quad$ a start state $\left(q_{0} \in Q\right)$

- $\quad$ a set of accept states $(F \subseteq Q)$

Now Q is set of states through which student's passes in the learning process. Each state defines knowledge level of a student in some particular topic. $\Sigma$ is finite set of input here input is Score of student which is calculated in each state depending upon Examination held on the state. Transition $\delta$ represents changing of knowledge or state. It is dependent on previous state and input on the state. The start state $q_{0}$ is the state when they have poor knowledge of the chapter. This process will be continued until student has sufficient knowledge about the subject and it is the accept state F. Score is calculated depending upon two parameters:

1) Accuracy (A).

2) Marks of the particular Examination (M)

$$
\mathrm{A}=\frac{\text { no of question corrected }}{\text { no of question attempted }} * 100
$$

$$
\begin{aligned}
\mathrm{M} & =\frac{\text { marks obtained }}{\text { total marks }} * 100 \\
\mathrm{~S} & =\frac{A+M}{2} \%
\end{aligned}
$$

Inputs are divided into three ranges $(\mathrm{R})$ according to $\mathrm{Score}(\mathrm{S})$.

$\mathrm{R}=\left\{\begin{array}{crr}1 & \text { if } & 0 \% \leq \mathrm{S} \leq 34 \% \\ 2 & \text { if } 35 \% \leq \mathrm{S} \leq 64 \% \\ 3 & \text { if } & \mathrm{S}>65 \%\end{array}\right\}$

Initial state $\mathrm{q}_{0}$ is the beginning of E-learning for a particular topic. In the initial state system first reviews student's curriculum, depth of knowledge by taking initial Examination. Then calculates the Score of the particular student based on that system decides next state of the student.
On each state it follows given activities Except Final and Initial state:

1) First activity is depending on their Score they are given study material, video of lectures for particular chapter. This decision can be taken either by teachers (if no previous case stored in case library) or previously stored cases are referred. Case Base Study (CBS) saves a lot of data for reference. It locates the solution method for the most similar case by comparing similarities. Based on Score retrieve the most similar case from the case library is a task for a CBS. CBS checks the Score and matches with previous cases. Out of these cases best one is selected and same study material, video of lectures given to the student. How to choose the case important factor of case evaluation will significantly impact on the completeness of the students learning and its assessment results. Selection can be decided by using Success Probability (SP).

SP for $\mathrm{i}$ th Case and $\mathrm{k}$ th input range is represented by $\mathrm{SP}_{\mathrm{ik}}=\frac{\sum_{j=1}^{n} S j}{n}$ where $\mathrm{n}$ is number of times the particular Case $C_{i}$ is used for $k$ th range $S_{j}$ is Score of particular student when the Case $\mathrm{C}_{\mathrm{i}}$ is used for $\mathrm{j}$ th time.

2) Second activity is to corrective assessment. this activity consists of two parts :

a) Solving queries and doubts of the students.

b) Problem-solving exercises given to them, it covers the same concepts and material as the first activity but asks questions in a slightly different way or format.

3) With previous step System also performs motivational activity it shows each time improvement of the student. In data base previous performance is stored state wise. By using the previous data performance analyzed for particular student.

4) After performing all above mentioned activities system takes an Examination, based on that Score will be calculated. This score decides transition of the state. But if student's performance reduces then study documents are re-verified according to students need.

When student have reached to the final state $F \subseteq Q$ that shows learning is complete for a particular topic. The same activity will be repeated for each chapter. Control flow diagram for each state activity is given below.

The following control flow diagram depicts the activity performed on each state. 


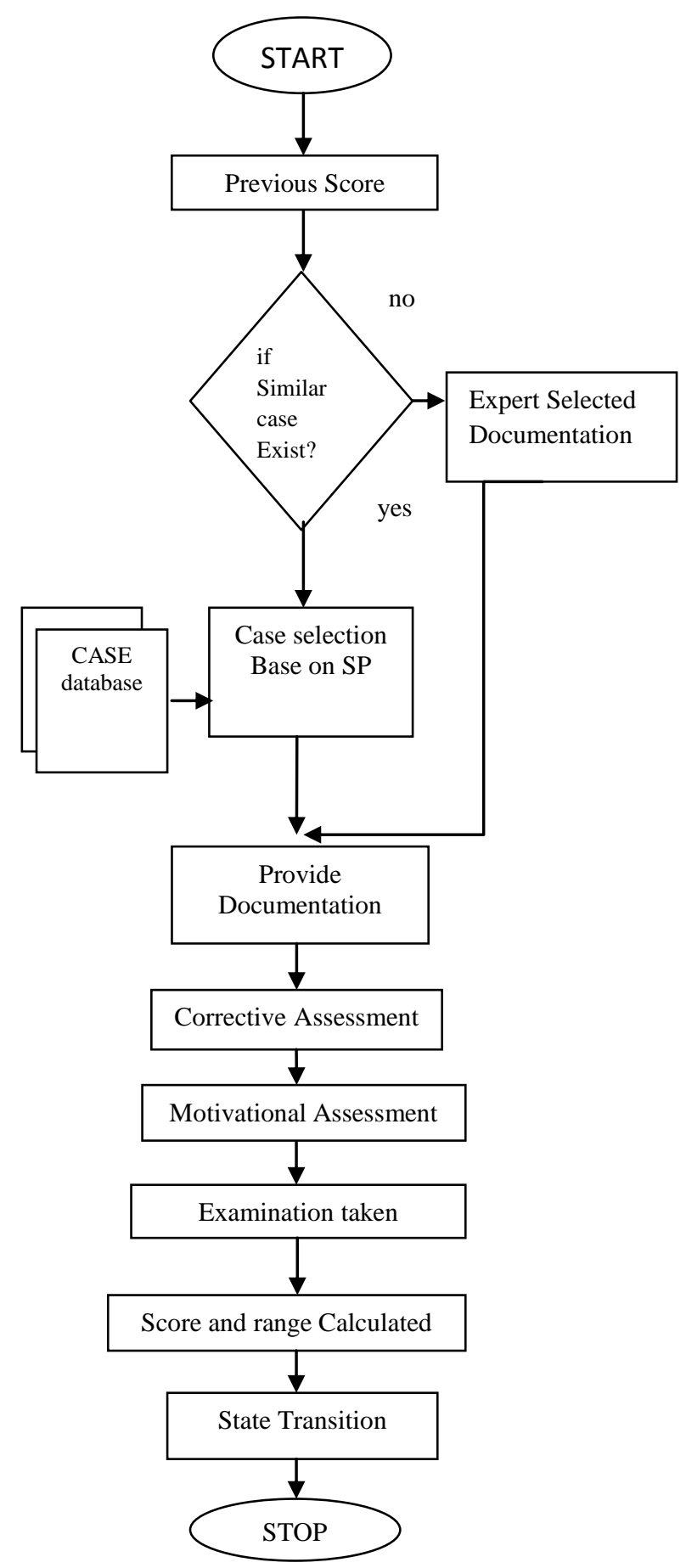

Fig 1 : Control flow diagram for activities followed on each state

\section{A TEST CASE WITH DFA}

In the following fig : 2 shows DFA for a E-leaning on any particular topic where $\mathrm{q}_{0}$ is initial state at this level, system takes one examination to identify depth of knowledge of a student in terms of Score by using formula 1, 2, 3. Then according to calculated score it determines the range given in formula 4 . Here range is the input to the state q0.

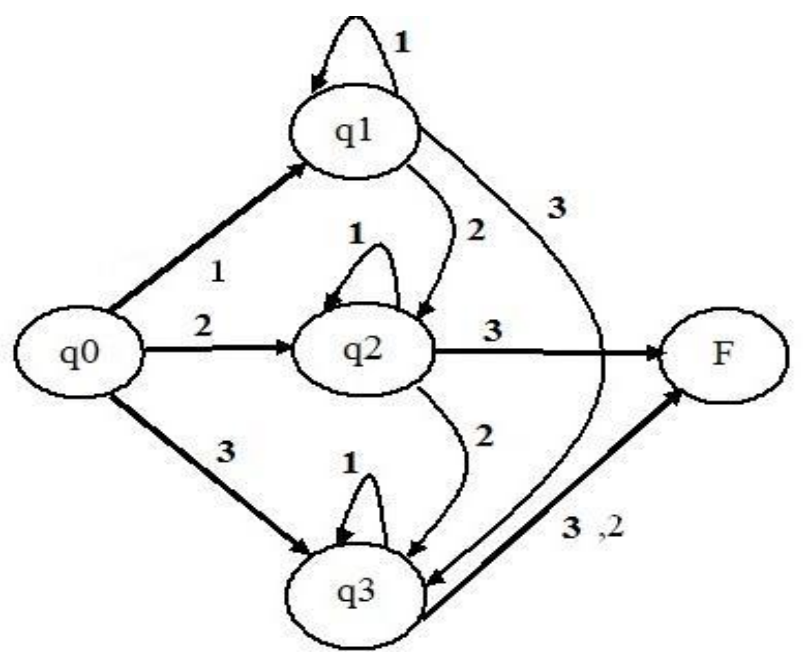

Fig 2: DFA for particular topic

The above state transition diagram work as follows:

Step1: if $\operatorname{input}\left(\mathrm{q}_{0}\right)=1$

goto state $\mathrm{q}_{1}$

Perform all the steps given in control flow diagram fig: 1 and calculate range, then repeat activity same as $\mathrm{q}_{0}$ according to state transition diagram given in fig: 2 until it reaches to the final state.

Step2: else if input $\left(\mathrm{q}_{0}\right)=2$

goto state $\mathrm{q}_{2}$

Perform all the steps given in control flow diagram fig: 1 and calculate range then repeat activity same as $\mathrm{q}_{0}$ according to state transition diagram given in fig: 2 until it reaches to the final state.

Step3: else goto state $\mathrm{q}_{3}$

Perform all the steps given in control flow diagram fig: 1 and calculate range then repeat activity same as $\mathrm{q}_{0}$ according to state transition diagram given in fig: 2 until it reaches to the final state

\section{CONCLUSION AND FUTURE SCOPE}

This paper improves adaptively nature of the e-learning system using Deterministic Finite Automata. It provides different level of effort to the different students. This system also motivates the students for encouraging them. Each time it shows analysis of performance for each and every student. From a more theoretical standpoint, this work can be seen as a new take on Interactive adaptive system. Where the learning to a topic is gradually constructed and modified by multiple interacting states. Here main goal is to give in depth knowledge to the student for each topic then they can move on next topic.

Sequencing of the curriculum is done manually by the expert of the subject. The idea of curriculum sequencing is to generate an individualized course for each student by dynamically selecting can be done in future. 


\section{REFERENCES}

[1] Herman Dwi Surjono "The Evaluation of a Moodle Based Adaptive e-Learning System" International Journal of Information and Education Technology, Vol. 4, No. 1, February 2014

[2] Dr. Latha Parthiban and Saranya Sekar"Ant Colony Optimization for Adaptive Learning "Indian Journal of Computational Intelligence \& Systems Sciences, Volume 1, Number 1, January - June 2013, pp. 19-24

[3] Mario Muñoz-Organero, Member, IEEE, Gustavo A. Ramírez, Pedro Muñoz Merino, and Carlos Delgado Kloos, Senior Member, IEEE " Analyzing Convergence in e-Learning Resource Filtering Based on ACO Techniques: A Case Study With Telecommunication Engineering Students" IEEE Transactions on Education, Vol. 53, No. 4, November 2010.

[4] Manju Bhaskar, Minu M Das, Dr. T. Chithralekha, Dr. S. Sivasatya "Genetic Algorithm Based Adaptive Learning Scheme Generation For Context Aware E-Learning" International Journal on Computer Science and Engineering Vol. 02, No. 04, 2010, 1271-1279

[5] Jose Manuel Marquez, Juan Antonio Ortega, Luis Gonzalez- "Creating adaptive learning paths using Ant
Colony" April, 2008, Francisco Velasco, IEEE Conference Publications.

[6] S. Gutierrez, G. Valigiani, Y. Jamont, P. Collet, and C. Delgado, "A Swarm Approach for Automatic Auditing of Pedagogical Planning," in Proc. IEEE Int. Conf. Adv. Learning Technol., Niigata, Japan, 2007,pp. 136-138.

[7] Marc El Alami, Nicolas Casel, and Denis Zampunieris, "An Architecture for e-Learning System with Computational Intelligence" 11th International Conference, KES 2007, XVII Italian Workshop on Neural Networks,2007,pg no.58-65.

[8] A. Dahbi, N. Elkamoun, A. Berraissoul"Adaptation and Optimization of Pedagogical paths by Ant's Algorithm." 2006 IEEE Information and Communication Technologies, ICTTA volume 1. pp. 546-551.

[9] Floriana Esposito, Oriana Licchelli, Giovanni Semeraro, "Discovering Student Models in e-learning Systems," Journal of Universal Computer Science, vol. 10, 2004,pg,no.47-57

[10] Semet, Y., Yamont, Y., Biojout, R., Luton, E. "Ant Colony Optimization for e-learning: Observing the emergence of pedagogic suggestions" IEEE Swarm Intelligence Symposium, 2003. 